\title{
Characteristics of the PTC Heater Used in Automotive HVAC Systems
}

\author{
Radu Musat and Elena Helerea \\ Department of Electrical Engineering, Faculty of Electrical Engineering and \\ Computer Science, Transilvania University of Brasov, \\ 29 Eroilor Str., 500036 Brasov, Romania \\ r_musat@yahoo.com, helerea@unitbv.ro
}

\begin{abstract}
The present heating method which uses the cooling system of the internal combustion engine of the vehicle takes a lot of time to heat the interior air. In order to improve the heating process, auxiliary devices are required. The new innovative techniques propose as auxiliary heating device positive temperature coefficient heaters. For a better control of the temperature, the parameters of these devices should be known. This paper deals with a detailed description of the new types of positive temperature coefficient heaters used in automotive field. The test bench system developed for determining the parameters and characteristics of the positive temperature coefficient heater is described. The obtained data of the thermal resistance and voltage-current characteristics are used for controlling the temperature in order to design and control the positive temperature coefficient heaters properly.
\end{abstract}

Keywords: PTC heater, HVAC, thermal comfort, vehicle, characteristics.

\section{Introduction}

The passenger's thermal comfort inside a vehicle is ensured by using heating, ventilating and air conditioning (HVAC) systems.

Modern internal combustion engines (ICE) with fossil fuels offer improved efficiency and dissipate less heat to the cooling system of the engine. On cold days, these engines produce little heat excess and are unable to provide the amount of heat for warming the vehicle interior up to a comfortable level [12].

In the automotive field, different technologies are available to provide the heat and to quickly ensure the passenger's thermal comfort. The conventional heating system uses the heat loss produced by ICE, which is transferred to the cooling system and then used for heating the vehicle interior air. This conventional heating method has disadvantages: it loses about $60 \%$ of the heat and the interior air temperature increases slowly. To improve the conventional heating method, auxiliary heating devices are required [16].

This paper describes and analyses an auxiliary heating device proposed to be used in the automotive field. Also, a test bench system is developed for determining the parameters and characteristics of the positive temperature coefficient (PTC) heater. 
The obtained data of the thermal-resistance $R=\mathrm{f}(T)$ and the voltage-current $U=\mathrm{f}(I)$ characteristics will be used for controlling the temperature in order to design the PTC heater for vehicle thermal comfort system.

\section{Contribution to Technological Innovation}

In the paper an innovative technique is proposed: a PTC heater is used as auxiliary heating device because of the specific thermal and electrical properties: thermal selfregulation, high responding time, quickly removing the condensed drops from the windscreen and improving the visibility through it.

Based on the experimental results described in the paper, a model and simulation of the PTC heater are proposed. An integration of the PTC model in the vehicle heating diagram for overall heating process simulation will be made in order to design and control optimally a PTC heater.

\section{State of the Art and Existing Problems}

Since the 1990's, research has been developed regarding the auxiliary heaters that warm up the interior air as soon as the vehicle is started [18-21]. There have been difficulties as regards the fact that the auxiliary heaters demand a large quantity of energy, and consequently they operate only when the engine is running, which increases the fuel consumption and the pollution.

After the significant increase in the fuel prices in 2006, much automotive research has shown renewed interest in fuel-efficient vehicles. In gasoline-fuelled vehicles, about $40 \%$ of fuel energy is wasted in exhaust heat, while 30\% of energy is transferred through the engine coolant. Because of that, these engines produce little heat excess, especially in winter time, in city traffic and traffic jams, and they are unable to warm quickly the passenger compartment to a comfortable level [12].

Various vehicle models from leading manufacturers around the world are now equipped with PTC auxiliary heaters. In 2007, 65\% of all diesel vehicles in Europe were equipped with an auxiliary heater and this is expected to rise to $90 \%$ by 2010 . It is obvious that PTC heaters will not be limited to diesel vehicles but will be also used in gasoline-powered ones as well [13].

Commonly used PTC materials include high density polyethylene (filled with graphite), polymeric and titanate ceramic materials (which work by grain boundary effects). PTC heaters based on barium titanate ceramic $\left(\mathrm{BaTiO}_{3}\right)$, having high resistance and power-dissipation characteristics, are mostly used in automotive industry [1], [2], [5], [7], [8].

Several authors have studied the resistance - temperature dependency of PTC heaters [3], [5], [6], [11], [15]. Over the years, various theoretical and experimental models have been developed to describe the PTC heater effect, e.g. in semiconducting $\mathrm{BaTiO}_{3}$, such as Heywang (the most accepted model), Jonker and Wang [4], [6], [7].

A comparative analysis between PTC thermistors and heaters sustains the development of new heating solutions. 


\section{PTC Thermistors and Heaters}

PTC thermistors have specific features: thermal self-regulating and efficient energy consumption, no danger of fire since there are no glowing parts, no excess temperature protection required, no smell, no radiation and oxidation, fast thermal response time, temperature setting from $+50{ }^{\circ} \mathrm{C}$ to $+320{ }^{\circ} \mathrm{C}$, compact design and long life service [10].

PTC thermistors have a wide variety of applications, which can be divided into two distinct categories [22]. The first category utilizes the resistance-temperature or voltage-current characteristics of the PTC thermistor. These are known as self heated applications and include: self-regulating heaters, PTC over-current protectors, time delay, motor starting etc. The second category comprises zero power or sensing applications (e.g. temperature sensors and control) [17].

The PTC thermistor has a low value of electrical resistance at low temperatures. When a voltage is applied to the cold elements, a high current is generated and the value of resistance rises with the temperature. As soon as the current flows through the device, the elements warm up by electrical dissipation until a steady state is reached and the resistive elements have reached their working temperature. This triggers the reduction of the electric current because the electric charge is unable to cross the boundaries of the tiny crystals at high temperatures. For this reason, the manufacturers of auxiliary heaters propose the use of the PTC thermistor principle [18], [19], [20], [21].

The PTC heater is mounted inside the HVAC system, after the heat exchanger. In Fig. 1, there is illustrated the position of the PTC heater and the air flow inside the HVAC system.

The PTC heater is connected to the vehicle electrical system and it is controlled by the Engine Control Module (ECM), HVAC control panel and relays [9]. In Fig. 2 there is illustrated the PTC heater control diagram.

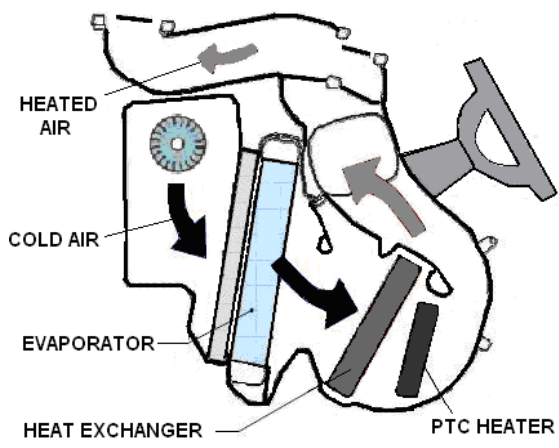

Fig. 1. HVAC system

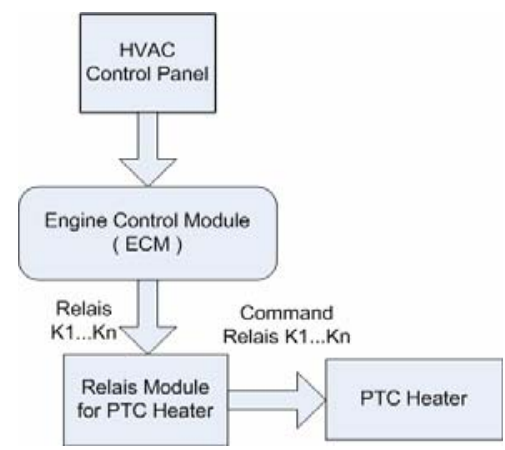

Fig. 2. PTC heater control diagram 
The PTC heater, illustrated in Fig. 3, consists of a moulded plastic plate (1), an electrical connector (2) and heating resistive elements with fins (3). The PTC heating resistive elements (Fig. 4) consist of small metallised ceramic plates (1), which are layered alternately along the unit core with aluminium radiator elements (2). These layers are held together by spring elements in a frame. The aluminium elements ensure the electrical contacts and transfer the heat to the passing heater air flow [14].

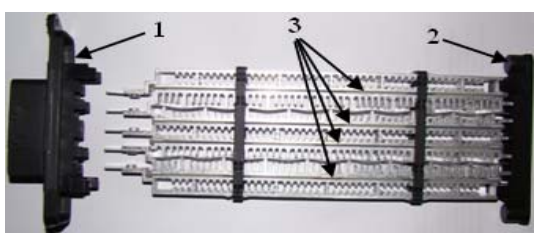

Fig. 3. PTC heater components

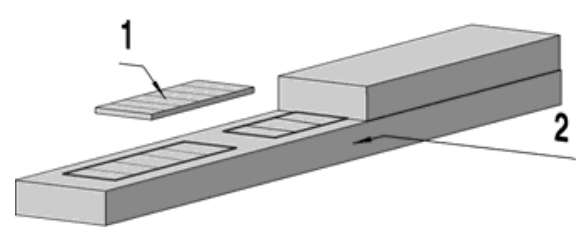

Fig. 4. PTC heating resistive elements

The heating resistive elements are separated in different heating circuits in order to adapt the heating power to different requirements and they act as positive temperature coefficient resistors. The maximum value of the temperature of the elements is around $165^{\circ} \mathrm{C}$, if no air flows through the heating system [12].

The heating elements are switched progressively in order to reduce the demand factor of the generator, to prevent load dumps and ECM problems caused by high currents.

The PTC heater operates only: (i) at low external temperatures; (ii) when insufficient heat is supplied by ICE cooling system; (iii) at reduced loads of the generator.

The total electrical power consumption for a PTC heater ranges between $900 \mathrm{~W}$ and $2000 \mathrm{~W}$, depending on the air volume of the vehicle interior. Vehicles equipped with PTC heater are fitted with a more powerful generator.

\section{Experiments and Results}

In order to model and simulate the processes which take place in the HVAC system, data related to the characteristics of the auxiliary heating devices are required. For a PTC heater, it is important to analyze and measure the temperature - resistance characteristics and the voltage-current characteristic in order to design the PTC heater and to determine the optimal thermal comfort.

To measure and obtain the temperature-resistance curves, $R=\mathrm{f}(T)$, a test bench was developed. The test bench, illustrated in Fig. 5, consists of a heating camera (type Carbolite PF/200), a cooling camera (type Derby DK 9620), temperature sensors (placed inside the heating/cooling camera) and a data acquisition system.

To measure and obtain the voltage-current characteristics $U=\mathrm{f}(I)$, a test bench was also developed. The test bench, illustrated in Fig. 6, consists of a battery (type Rombat Pilot Diesel Hybrid, 12V / 77Ah), an ammeter, a voltmeter, temperature sensors (placed on PTC heating elements) and a data acquisition system.

All the acquired data are processed by means of MATLAB tool. 


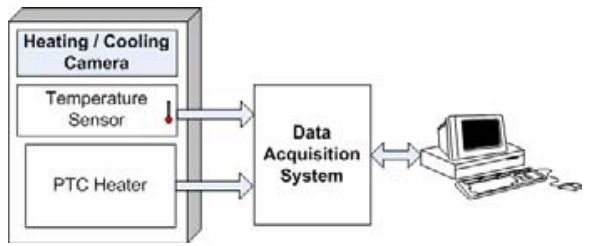

Fig. 5. Test bench scheme implementation for thermal resistance characteristics

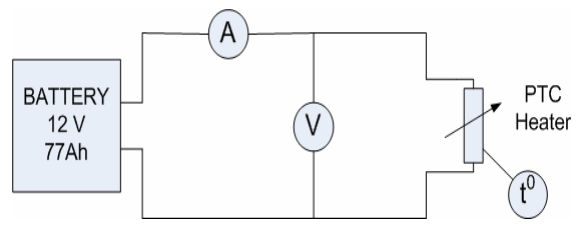

Fig. 6. Measurement scheme for determining $U=\mathrm{f}(I)$ characteristics

To make the experiments, a $1000 \mathrm{~W} / 13.5 \mathrm{~V}$ PTC heater sample was used. This heater consists of four power stages (two stages of $333 \mathrm{~W}$ and two stages of $166 \mathrm{~W}$ ). In Fig. 7, there is illustrated the electrical diagram for the PTC heater sample.

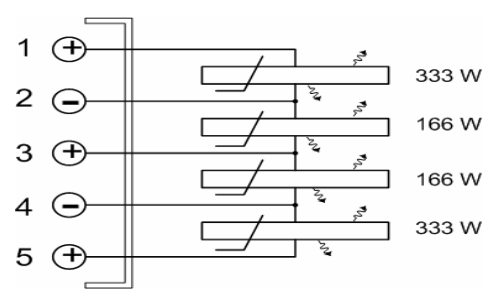

Fig. 7. Electrical diagram of the PTC heater sample

In Fig. 8 and Fig. 9, there are illustrated the experimental results representing the characteristics $R=\mathrm{f}(T)$ and $U=\mathrm{f}(I)$, for each stage.

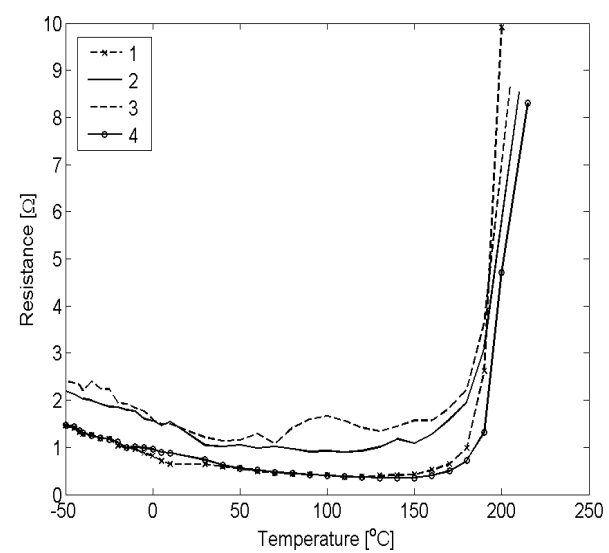

Fig. 8. Thermal resistance characteristics

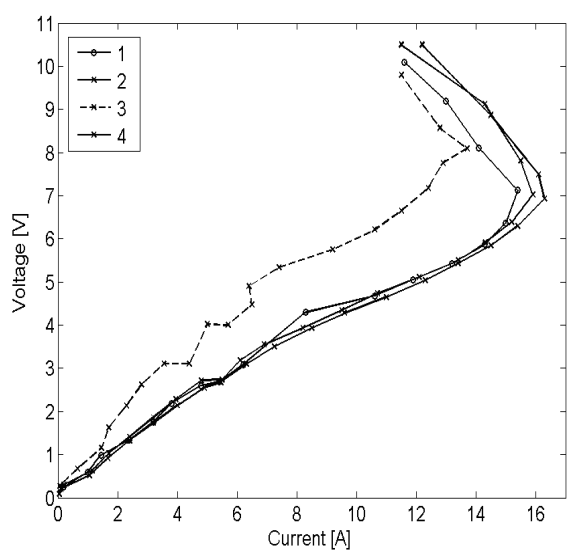

Fig. 9. Voltage-current characteristics 
As it can be seen in Fig. 8, the obtained data show that the resistance initially decreases with the increase in temperature. In the low temperature region, the resistance curve has a negative temperature coefficient. The minimal values of the resistance obtained at specific threshold temperatures, $T_{\mathrm{t}}$ and the calculated values for the temperature coefficient of the resistance $\alpha_{R}$ (for the negative and positive slope), for each stage are given in Table 1. For temperatures greater than $T_{\mathrm{t}}$, the negative temperature coefficient is changed to a positive one.

Table 1. The values of the resistance $R$, the threshold temperatures $T_{\mathrm{t}}$. and the temperature coefficient of the resistance $\alpha_{R}$ for each stage.

\begin{tabular}{ccccc}
\hline Stage & $R[\Omega]$ & $T_{\mathrm{t}}\left[{ }^{\circ} \mathrm{C}\right]$ & $\alpha_{\mathrm{R}-}\left[\mathrm{K}^{-1}\right]$ & $\alpha_{\mathrm{R}+}\left[\mathrm{K}^{-1}\right]$ \\
\hline 1 & 0.367 & 120 & -0.0044 & +0.937 \\
2 & 0.895 & 110 & -0.0037 & +0.285 \\
3 & 1.34 & 130 & -0.0024 & +0.211 \\
4 & 0.35 & 130 & -0.0042 & +0.984 \\
\hline
\end{tabular}

The values of the temperature coefficient of resistance require specific thermal regulating process characteristics of the PTC heater. Based on these coefficients, the PTC heaters can be properly designed. The thermal resistance characteristic is appropriate for preventing the PTC heater overheating.

As it is shown in Fig. 9, the voltage-current characteristics are linear until the PTC heating elements have reached their working temperature and a steady state is reached. After these values, the electrical current drastically decreases.

Warming up of the PTC heater depends on the electrical power inside the elements and can be described with the following relationship:

$$
U(I) \cdot I(t)=\frac{U^{2}(t)}{R(T, t)} \cdot \delta \cdot\left(T-T_{A}\right)+C_{P T C} \cdot \frac{d T}{d t} .
$$

where:

$U(t)$ - voltage supply; $I(t)$ - electrical current through the PTC heater; $\delta$ - heat dissipation of PTC; $T$ - present temperature of PTC; $T_{\mathrm{A}}-$ ambient temperature; $\mathrm{C}_{\mathrm{PTC}}-$ heat capacity of PTC; $\mathrm{d} T / \mathrm{d} t$ - time temperature variation.

Based on the voltage and current data values, the dissipation power - temperature characteristics for each stage are obtained and illustrated in Fig. 10.

As it can be seen in Fig. 10, there is a maximum dissipation power corresponding to the threshold temperatures for each PTC heater power stage.

The obtained values of the parameters and the characteristics can be compared with those from specialty literature. The PTC heater tested above proved to have the same characteristics with those from a typical PTC thermistor model. The developed test bench allows testing these characteristics rapidly, efficiently and accurately.

The high responding time is the typical feature of the PTC heater. This is useful after the vehicle is started: till the engine reaches its operating temperature, the conventional heating system does not supply the necessary heat for the interior air. This additional heat can practically be accounted to the PTC heater. 


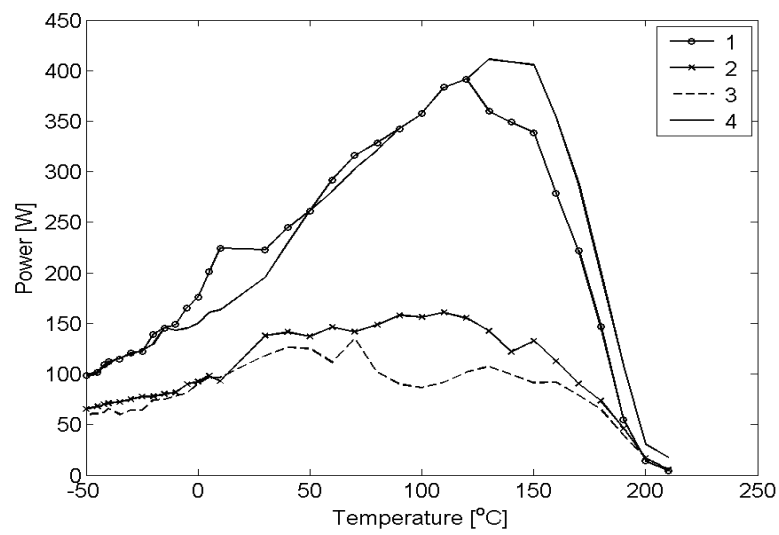

Fig. 10. Dissipation power - temperature characteristics for PTC heater sample

\section{Conclusion and Future Work}

This paper deals with an analysis on auxiliary heating devices used in vehicle thermal comfort systems. A test bench system was developed for determining the parameters and characteristics of PTC heater and measurement data were processed.

Based on the experimental results described in the paper, a model and simulation of the PTC heater was developed. An integration of the PTC model in the vehicle heating diagram for overall heating process simulation is to be made.

The PTC heaters are necessary in some conditions (e.g. winter time) to increase the passenger's thermal comfort. The PTC heaters are ideal for thermal comfort control due to their capacity of thermal self-regulating and high responding time.

The challenge is that the PTC heater demands a large quantity of electrical current, so it operates only when the engine is running and thus increases the fuel consumption and the pollution.

New higher capacities of PTC heater would be possible with a $42 \mathrm{~V}$ vehicle wiring system. The transition to $42 \mathrm{~V}$ voltages, the decentralisation of power distribution and the development of more complex energy sources will provide new innovative systems to supply PTC heaters properly.

Since vehicles are getting more efficient, they free less heat for the heating system; thus, by including auxiliary devices consuming electric energy, the heating time will be improved and the engine efficiency increased. The electric energy is taken from the engine through the generator/battery system, which reduces the overall efficiency of the vehicle. Consequently, an adequate energy balance and management should be accomplished.

Increasing the overall efficiency is the main objective in any PTC heater design and can be achieved by different techniques, such as: improving the technology of materials, manufacture and control processes. 


\section{References}

1. Amin, A.: Piezoresistivity in semiconducting positive temperature coefficient ceramics. Journal of the American Ceramic Society 72(3), 369-376 (1989)

2. Heywang, W.: Bariumtitanat als Sperrschichthalbleiter. Solid-State Electronics 3, 51-58 (1961)

3. Heywang, W.: Resistivity anomaly in doped barium titanate. Journal of the American Ceramic Society 47(10), 484-490 (1964)

4. Heywang, W.: Semiconducting barium titanate. Journal of Materials Science 6, 1214-1224 (1971)

5. Huybrechts, B., Ishizaki, H.: The positive temperature coefficient of resistivity in barium titanate. Journal of Materials Science 30, 2463-2474 (1995)

6. Jonker, G.H.: Some aspects of semiconducting barium titanate. Solid-State Electronics 7, 895-903 (1964)

7. Wang, D.Y.: Electrical properties of PTC barium titanate. Journal of the American Ceramic Society 73(3), 669-677 (1990)

8. Mamunya, Y.P., Muzychenko, Y.V.: PTC effect and structure of polymer composite. Journal of Polymer Engineering and Science 47, 34-42 (2007)

9. Amsel, C., Schoenen, R.: Use of CAE-Methods to analyse the influence of new electrical systems behaviour on tomorrow's 42V. Institut für Kraftfahrwesen, Aachen (2001)

10. Jones, L., Kinsman, K.: PTC Thermistor Introduction. Compliance Engineering Magazine (2002)

11. Schedel, R.: New PTC Heaters Control High Voltages in Hybrid and Electric Vehicles. Eberspaecher Magazine (2009)

12. Daly, S.: Automotive air conditioning and climate control systems. Elsevier, Oxford (2006)

13. Ehsani, M., Gao, Y.: Modern Electric, Hybrid Electric, and Fuel Cell Vehicles - Fundamentals, Theory, and Design. CRC Press, Washington (2005)

14. Stone, R.: Introduction to Internal Combustion Engines, 3rd edn. SAE International and MacMillan Press, USA (1999)

15. Apostol, I., Helerea, E.: Obtaining the High Performance PTC Thermistors based on Barium Titanate. In: Proceeding of the 11th International Conference on Optimization of Electric and Electronic Equipment - Optim. 2008, Brasov, pp. 119-123 (2008)

16. Kassakian, J.G., Perreault, D.J.: The Future of Electronics in Automobiles. In: Proceeding of ISPSD, pp. 15-19. IEEE Explore, Los Alamitos (2001)

17. Spectrum Sensors \& Control Inc., Advanced Thermal Products Division, http: / /www. specsensors.com

18. Denso Corporation, http://www.globaldenso.com

19. Behr Hella Service GmbH, http: / / www . behrhellaservice.com

20. Valeo Termico SA, http: / / www . valeo.com

21. Eberspaecher electronics GmbH, http: / /www. eberspaecher.com

22. Advanced thermal Products Inc., http://www. atpsensor.com 